\title{
Grand challenges for cancer imaging and diagnosis
}

\section{Giuseppe Esposito*}

Department of Radiology, Georgetown University Hospital, Washington, DC, USA

${ }^{*}$ Correspondence: exg11@gunet.georgetown.edu

In the last two decades, the field of cancer imaging has witnessed tremendous evolution which has impacted virtually every aspect of research and clinical management of cancer. This evolution has been the result of innovations in two main aspects: 1. Instrumentation and 2. Development of new contrast agents and radiolabeled tracers. Among the most significant innovations in instrumentation, the introduction of "hybrid" scanners such as PET/CT (Townsend et al., 2004) has provided new tools for the characterization, staging, and restaging of many types of cancer. New more sensitive and faster scanners, new hybrid combinations such as PET/MRI are already available, and new contrast agents and radiolabeled tracers for MRI, ultrasound, PET/SPECT, and optical imaging are being developed. The evolution toward more sensitive "hybrid" scanners and of new imaging agents has led to the growth of the field of Molecular Imaging which, as defined by the Molecular Imaging Center of the Society of Nuclear Medicine is "The visualization, characterization, and measurement of biological processes at the molecular and cellular levels in humans and other living systems." It is particularly important that progress in cancer imaging continues, to parallel the advancements in the understanding of the molecular and cellular processes of cancer transformation. Translational research on cancer biology can benefit from the contribution of molecular imaging to produce valuable diagnostic and prognostic in vivo biomarkers to support and improve the discovery, development, and testing of new therapeutic tools, alone or in combination with existing treatment modalities. The final product of this process would be that for each patient there is a personalized biological characterization of cancer leading to individualized treatment and assessment of tumor response. Existing clinical trials have already included molecular imaging and specifically PET/CT in the algorithm to assess response to treatment earlier and more accurately. For example, CALGB 50804 and 50604 phase II clinical trials for non-bulky and bulky Hodgkin lymphoma use PET/CT to provide an early assessment of treatment response after two cycles of chemotherapy in order to guide responseadapted individualized patient treatment. Patients with a negative PET/CT scan are classified as early responders and continue on the same chemotherapy regimen, whereas non-responders are switched to a different chemotherapy with or without radiation therapy. In solid tumors, the MUNICON phase II trial (Lordick et al., 2007) uses PET/CT imaging for an early assessment of response after two cycles of neoadjuvant chemotherapy in patients with esophageal cancer. Responders receive the full course of chemotherapy whereas non-responders will go directly to surgery without additional cycles of neoadjuvant chemotherapy.

As cancer imaging is moving toward the goal to provide reliable and sensitive markers for tumor diagnosis and assessment of response to treatment, the limitations and challenges of this field become however more apparent and new questions are raised, not only for the new imaging techniques and tracers under development, but also for the more established imaging modalities. As progress is being made, we need to turn back to the techniques and guidelines in current use and ask ourselves if we can do better. Are RECIST (Therasse et al., 2000; Eisenhauer et al., 2009) criteria for response assessment in solid tumors adequate, or should we use a volumetric assessment of tumor size? Do we need 3D measurements of tumor volumes and if so, do we have adequate software for a routine 3D measurement of tumor volumes in standard clinical care or in clinical trials? As we learn more on tumor biology through FDG-PET imaging, we realize the limitations of size criteria for response assessment (for review see Weber, 2009), and understand that a combined structural and functional assessment offers a more complete and probably more reliable evaluation of treatment response. Our challenge is to understand how to best use CT based structural information and functional PET information to assess tumor response. We have learnt that FDG-PET significantly improves staging and restaging of most cancers, but we still do not know how to best define tumor response based on FDG uptake, or how to best measure FDG uptake. It is not fully understood how and when to best use the FDG-PET for response assessment and tumor surveillance. Most importantly however our biggest challenge is to improve the reproducibility of the functional information offered by molecular imaging so that we can best relate this information to established disease outcome measures through well designed image based clinical trials.

Frontiers in Cancer Imaging and Diagnosis welcomes technical and clinical papers that address the challenges and limitations of current techniques, or that present new findings and potential use of these techniques, with the final goal to optimize their use. Studies that compare different imaging modalities or that show the complementary role multimodality imaging are believed to be important to address the challenges on how to best utilize the current imaging modalities.

The world of cancer imaging is blessed by a vibrant community of scientists that work to create contrast agents and tracers for different aspects of cancer biology: DNA synthesis and cellular proliferation (Vesselle et al., 2002), tumor hypoxia (Dubois et al., 2004), angiogenesis and apoptosis (Beer and Schwaiger, 2008; Belhocine, 2008), glucose metabolism to cite only some. Many of these new agents have already been tested in humans and in clinical trials, many are being evaluated in animal imaging trials. Frontiers in Cancer Imaging and Diagnosis recognizes the challenges that are associated with studies that evaluate the feasibility of new imaging modalities, and with the 
use of these new agents to explore the effect of cancer treatment on different aspects of tumor biology, and welcomes publications in this field. These studies will eventually lead to a more individualized assessment of tumor biology and personalized treatments.

Progress in cancer imaging can also guide toward a more personalized and potentially more effective use of image guided interventions. As a number of image guided interventions are available (focused radiation treatment, embolization, chemoembolization, radiofrequency ablation, radiochemoembolization etc.), we realize that some of these interventions are more successful for some types/ subtypes of tumors or biological cancer conditions, and less successful in others. It is conceivable for example, that in conditions of hypoxia, tumors may be more resistant to treatment (Eschmann et al., 2005), and it is conceivable that for instance imaging of tumor hypoxia could direct the type and timing of local interventions in coordination with systemic treatments. The challenges ahead are not only in the production of the imaging agents and choice of best imaging modality, but also in the design and implementation of appropriate translational trials to apply the information obtained from imaging to the type of treatment and patient outcomes. Frontiers in Cancer Imaging and Diagnosis welcomes publication of studies from the initial phases of implementation of new imaging techniques, all the way to studies that explore the role of molecular imaging during patient treatment.

There is no question that the ever evolving field of Cancer Imaging remains a central portion in the research and clinical management of cancer, and there is no question that although many challenges remain ahead, this field will contribute to continued improvement in the detection of the disease and to more effective treatments.

\section{REFERENCES}

Beer, A. J., and Schwaiger, M. (2008). "Imaging of angiogenesis," in Molecular Imaging in Oncology, eds M. G. Pomper and J. Gelovani (New York, NY: Informa Healthcare), 469-491.

Belhocine, T. Z. (2008). "Imaging apoptosis," in Molecular Imaging in Oncology, eds M. G. Pomper and J.Gelovani (New York, NY: Informa Healthcare), 493-502.

Dubois, L., Landuyt, W., Haustermans, K., Dupont, P., Bormans, G., Vermaelen, P., Flamen, P., Verbeken, E., and Mortelmans, L. (2004). Evaluation of hypoxia in an experimental rat tumour model by [(18)F]fluoromisonidazole PET and immunohistochemistry. $\mathrm{Br}$. J. Cancer 91, 1947-1954.

Eisenhauer, E. A., Therasse, P., Bogaerts, J., Schwartz, L. H., Sargent, D., Ford, R., Dancey, J., Arbuck, S., Gwyther, S., Mooney, M., Rubinstein, L., Shankar, L., Dodd, L., Kaplan, R., Lacombe, D., and Verweij, J. (2009). New response evaluation criteria in solid tumours: revised RECIST guideline (version 1.1). Eur. J. Cancer $45,228-247$.

Eschmann, S. M., Paulsen, F., Reimold, M., Dittmann, H., Welz, S., Reischl, G., Machulla, H. J., and Bares, R. (2005). Prognostic impact of hypoxia imaging with 18F-misonidazole PET in non-small cell lung cancer and head and neck cancer before radiotherapy. J. Nucl. Med. 46, 253-260.
Lordick, F., Ott, K., Krause, B. J., Weber, W. A., Becker, K., Stein, H. J., Lorenzen, S., Schuster, T., Wieder, H., Herrmann, K., Bredenkamp, R., Höfler, H., Fink, U., Peschel, C., Schwaiger, M., and Siewert, J. R. (2007). PET to assess early metabolic response and to guide treatment of adenocarcinoma of the oesophagogastric junction: the MUNICON phase II trial. Lancet Oncol. 8, 797-805.

Therasse, P., Arbuck, S. G., Eisenhauer, E. A., Wanders, J., Kaplan, R. S., Rubinstein, L., Verweij, J., Van Glabbeke, M., van Oosterom, A. T., Christian, M.C., and Gwyther, S. G. (2000). New guidelines to evaluate the response to treatment in solid tumors. European Organization for Research and Treatment of Cancer, National Cancer Institute of the United States, National Cancer Institute of Canada. J. Natl. Cancer Inst. 92, 205-216.

Townsend, D. W., Carney, J. P. J., Yap, J. T., and Hall, N. C. (2004). PET/CT today and tomorrow. J. Nucl. Med. 45(Suppl.), 4S-14S.

Vesselle, H., Grierson, J., Muzi, M., Pugsley, J.M., Schmidt, R. A., Rabinowitz, P., Peterson, L. M., Vallieres, E., and Wood, D. E. (2002). In vivo validation of 3'deoxy-3'[(18)F]fluorothymidine ([(18)F]FLT) as a proliferation imaging tracer in humans: correlation of [(18) F]FLT uptake by positron emission tomography with Ki-67 immunohistochemistry and flow cytometry in human lung tumors. Clin. Cancer Res. 8, 3315-3323. Weber, W.A. (2009). Assessing tumor response to therapy. J. Nucl. Med. 50, 1S-10S.

Received:26March 2011; accepted:20 June 2011; published online: 05 July 2011.

Citation: Esposito G (2011) Grand challenges for cancer imaging and diagnosis. Front. Oncol. 1:15. doi: 10.3389/ fonc.2011.00015

This article was submitted to Frontiers in Cancer Imaging and Diagnosis, a specialty of Frontiers in Oncology.

Copyright $\odot 2011$ Esposito. This is an open-access article subject to a non-exclusive license between the authors and Frontiers Media SA, which permits use, distribution and reproduction in other forums, provided the original authors and source are credited and other Frontiers conditions are complied with. 